\title{
HCV drugs reduce transplantation need
}

Treatment with direct-acting antiviral agents (DAAs) in patients with decompensated cirrhosis caused by HCV infection could enable the removal of a fifth of these individuals from liver transplantation waiting lists, according to a new study.

The availability of safe and effective DAAs has transformed the treatment of patients infected with $\mathrm{HCV}$ within the past few years, yielding a sustained viriologic response in $>90 \%$ of patients with HCV-related compensated cirrhosis. However, the clinical benefit of these drugs in patients with more severe HCV-related decompensated cirrhosis who require liver transplantation was previously unknown.
Luca Saverio Belli and colleagues therefore carried out a multicentre retrospective study examining the effect of DAA treatment in 103 patients with decompensated HCV-related cirrhosis who were all initially listed for liver transplantation. The team found that DAA administration resulted in clinical improvement that merited waiting-list inactivation in one in three patients, with one in five patients delisted altogether following a substantial recovery. Furthermore, so-called inactivated patients showed an improvement in both Model for End-stage Liver Disease and Child-Pugh prognosis scoring systems and 16 patients experienced a complete regression in liver decompensation signs.
"DAA treatment and associated delisting could significantly reduce the need [for liver transplantation] ... and save organs," the researchers write in their discussion. The team also suggest that follow-up studies are now required to determine the long-term mortality in patients with

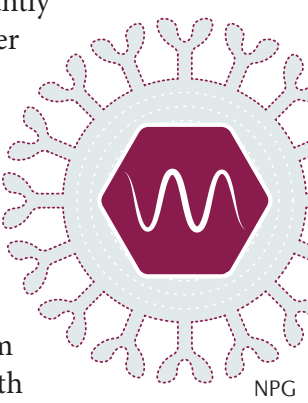
HCV-related decompensated cirrhosis who have received DAAs.

\section{Charlotte Ridler}

ORIGINAL ARTICLE Belli, L. S. et al. Delisting of liver transplant candidates with chrnic hepatitic C after viral eradication: a European study.J. Hepatol. http://dx.doi.org/10.1016/j.jhep.2016.05.010 (2016) 